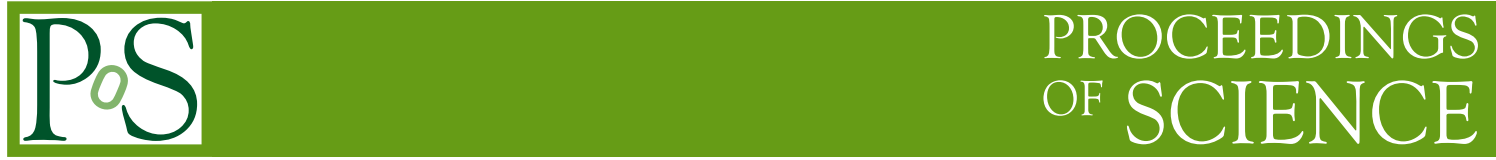

\title{
PHENIX plans for RHIC low energy run
}

\section{Takao Sakaguchi*, for the PHENIX collaboration}

Physics Department, Brookhaven National Laboratory

E-mail: takao@bnl.gov

PHENIX plans for low energy running are presented. Current detector setting makes it possible to measure dielectron spectra down to $\sqrt{s_{N N}}=39 \mathrm{GeV}$, and photon/high $p_{T}$ hadron spectra down to below sub-injection energy $\left(\sqrt{s_{N N}}=5-10 \mathrm{GeV}\right)$. The upgrade of the trigger scheme after the installation of VTX detector will enable PHENIX to fully explore the sub-injection energy regime, starting 2011.

5th International Workshop on Critical Point and Onset of Deconfinement - CPOD 2009,

June 08 - 122009

Brookhaven National Laboratory, Long Island, New York, USA

\footnotetext{
${ }^{*}$ Speaker.
} 


\section{Introduction}

Since the start of RHIC running in 2000, many discoveries have been made at RHIC not only in conventional observables that have been measured at lower energies but also in new phenomena that were predicted but have never been observed [1, 2, 3, 4]. Figure 1(a) shows the cartoon of a prediction, drawn by Shoji Nagamiya, the first spokesperson of the PHENIX collaboration, on what is expected for observables already found in low $\sqrt{s}$ experiments, when we cross "the" boundary between hadron and quark-gluon phases. At RHIC, most of these observables have
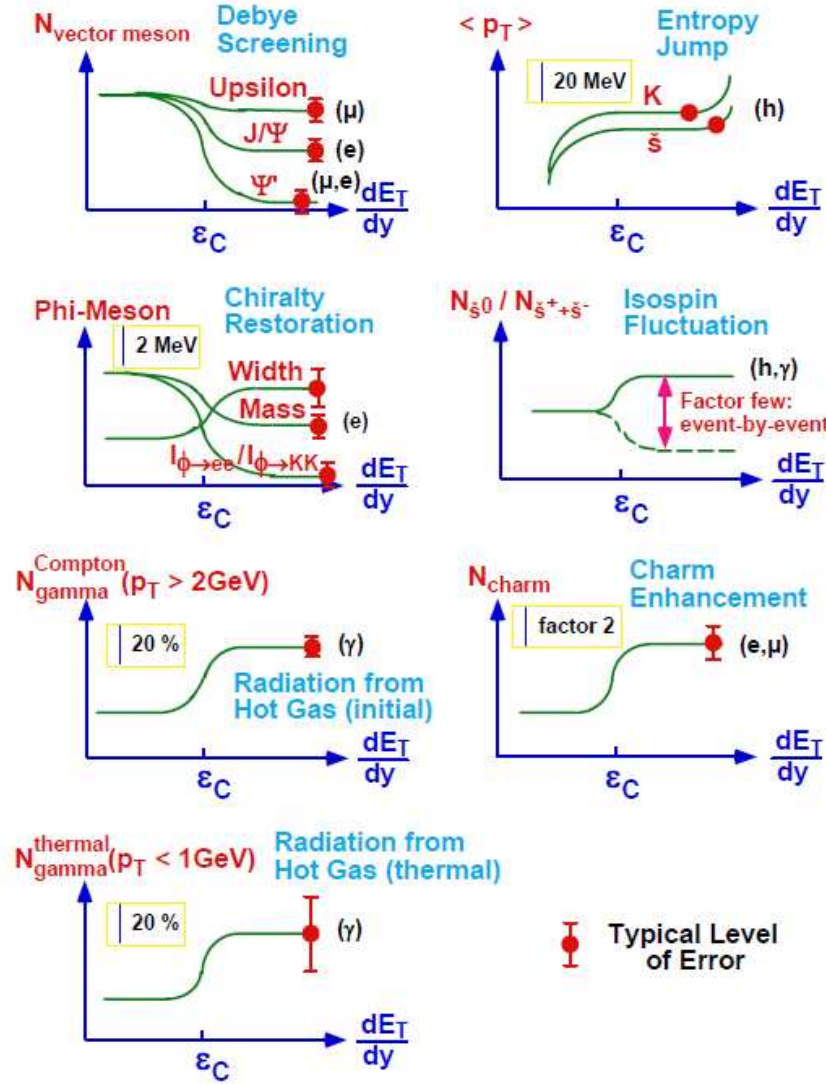

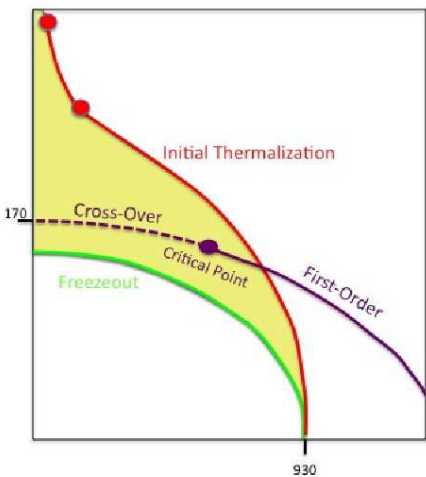

Good

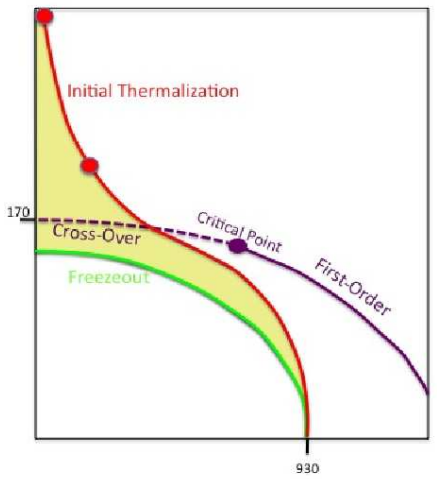

Bad

Figure 1: Left: (a) expectation on crossing "the" QCD phase transition. Right: (b) Possible locations of critical points relative to freezeout and thermalization lines.

been measured, however, no critical behavior has been seen in them so far. On the other hand, several new phenomena have been discovered at RHIC, such as jet quenching or prompt production, relying on hard scattering process, whose cross-section has increased drastically at this high $\sqrt{s}$. High transverse momentum $\left(p_{T}\right)$ particle correlations ( $\gamma$-hadron and dihadron correlations) that are also produced in the initial hard scattering were also measured at RHIC for the first time. The RHIC results triggered a new look of SPS data, and indeed many of these probes were found to have existed already at SPS energies, however, these results were published only after the discovery has been made at RHIC. This is an example how a high $\sqrt{s}$ machine opened up a new field. It tells that the low energy running at RHIC is not a repetition of exercises done in the past, but will study 
new phenomena through new probes inaccessible so far due to insufficient statistics. Therefore, the low energy running at RHIC is old in the sense of $\sqrt{s}$, but is new in the sense that we are going to look at new probes.

There have been many studies on where the critical end point is located. The question is whether or not this location is in between initial thermalization and freezeout on QCD phase diagram. If not, no experiment may see the signature of critical point (Fig. 1(b)). Obviously, the search for the critical point is not a problem dominated either by experiment or theory alone. A close collaboration between experimentalists and theorists is essential.

\section{Issues in low energy running}

Measurement at lower $\sqrt{s}$ should be relatively easy in terms of multiplicity or particle identification (lower $\sqrt{s}$ system would not produce as many and high energy particles as $\sqrt{s}=200 \mathrm{GeV}$ did). However, since the detector systems are optimized to measure these probes and took some advantages from phenomena that are realized at higher $\sqrt{s}$, running the detector at lower $\sqrt{s}$ may require addition of a dedicated device. The most crucial part is triggering and characterizing events. Because of very low multiplicity, it is possible that the trigger becomes less efficient. Moreover the particles with high rapidity are dominantly produced by fragmentation process, and thus the magnitude of the particle multiplicity is no longer an excellent measure of collision centrality. PHOBOS experiment at RHIC has measured pseudo-rapidity distributions in $\mathrm{Cu}+\mathrm{Cu}$ at various $\sqrt{s}$ (Fig. 2) [5]. As is seen from the plot, at $3.1<|\eta|<3.9$ where the beam-beam counter (BBC) of the PHENIX detector is installed, the multiplicity is very low even at $\sqrt{s}=22.4 \mathrm{GeV}$ [6]. The smaller number of detected particles will also result in degrading of timing resolution, and thus the vertex position resolution becomes poorer. PHENIX installed the reaction-plane (RxNP) detector
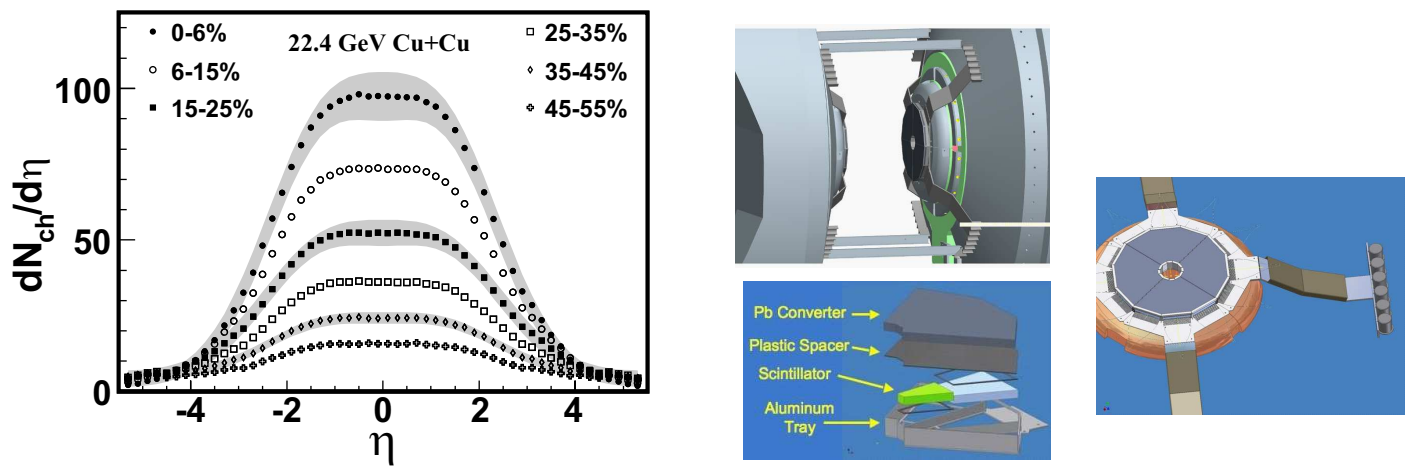

Figure 2: Left: Charge multiplicity measurement at $\sqrt{s_{N N}}=22.4 \mathrm{GeV}$ performed by PHOBOS experiment. Right: PHENIX RxNP detector.

that covers $1<|\eta|<2.8$ before starting Run-7. It is for determining the reaction plane with a better resolution than the one with the BBC. Since the multiplicity at this rapidity region is higher than at $3.1<|\eta|<3.9$ (BBC acceptance), it would give a complementary information for triggering.

Another issue at the low energies (around sub-injection energy) is rejection of events not related to physics collisions, namely, beam-gas interactions, beam scrape, and beam-beam interactions outside the vertex selections. PHENIX is going to install a silicon vertex detector covering 
$|\eta|<1.2$ in 2011 , and has a plan to make use of the signal from the detector to reject such events at even lower energy such as sub-injection energy (5-10 GeV) (Fig. 3). As a result of these im-
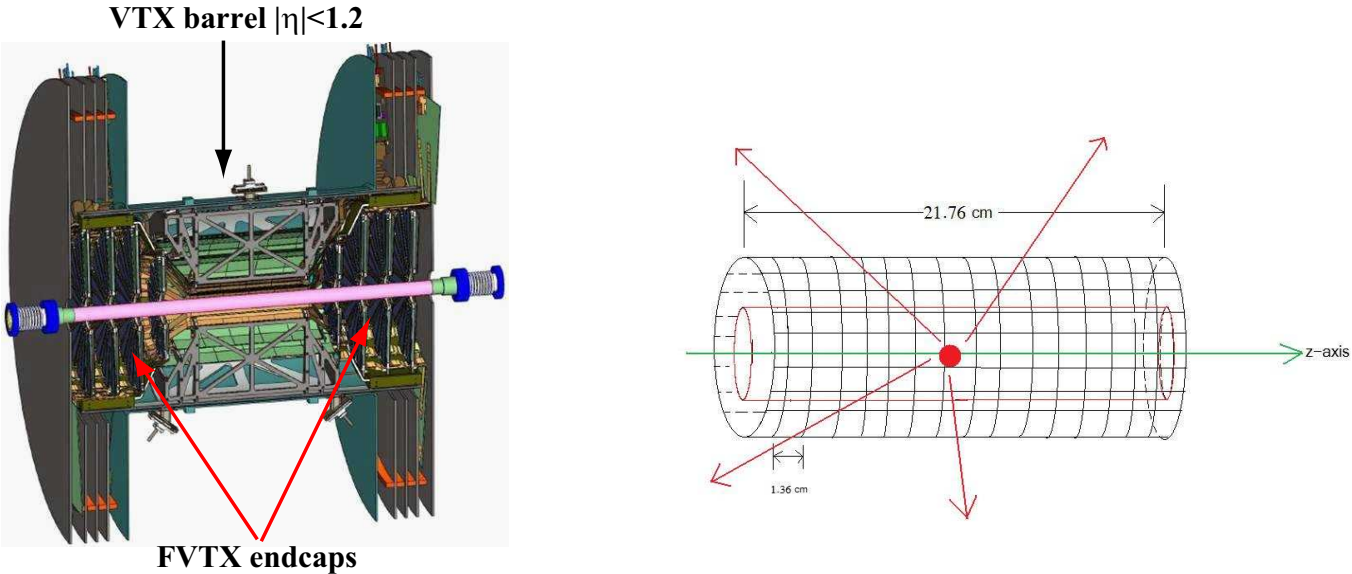

Figure 3: Left: VTX detector. Right: trigger map using signals from VTX detector. The signals from each $\mathrm{pad} / \mathrm{strip}$ will be summed and sent to global trigger system.

provements, PHENIX will be fully efficient in triggering and characterizing events at low $\sqrt{s}$. The expected acceptance of the PHENIX detector by then is shown in Fig. 4. Before 2011, the VTX de-

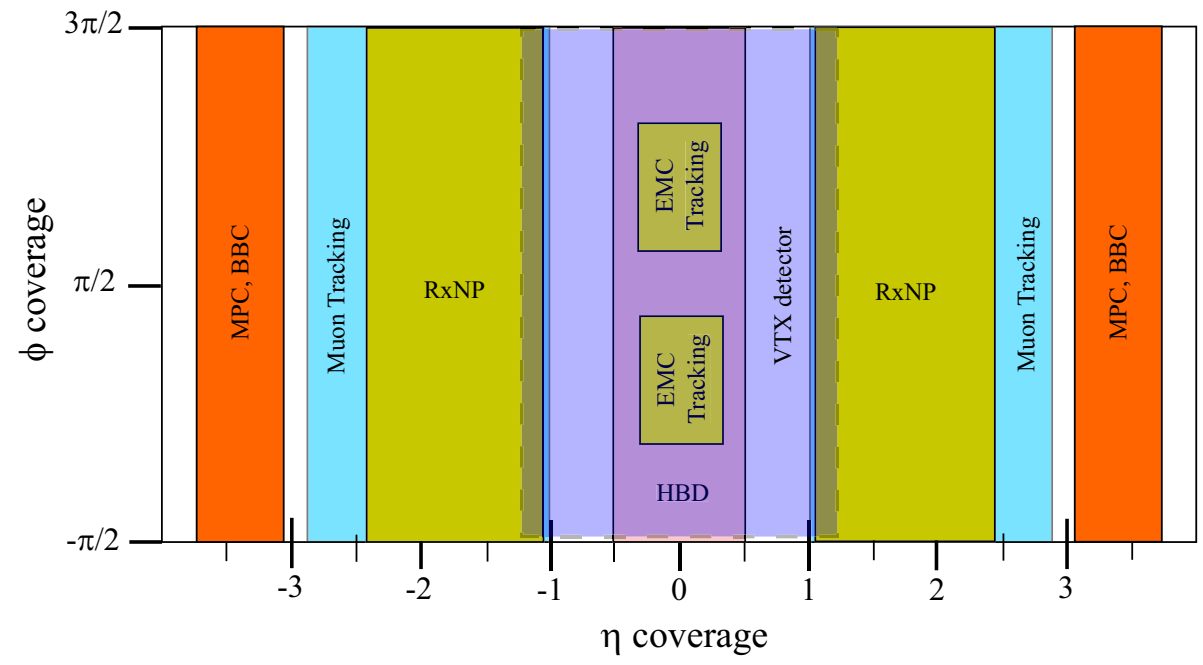

Figure 4: Acceptance of the PHENIX detector after 2011. HBD is installed now, as shown in the schematics, but will be taken off in accordance with the installation of VTX detector.

tector will not be installed because the same space is occupied by a hadron blind detector (HBD) [7] which targets a precise dielectron measurement in full energy Au+Au running in 2010. The HBD can be operated in non-hadron blind mode, and positively detect charged particles. However, it can not detect vertex points of the particles. 


\section{Rich Physics with the PHENIX detector}

PHENIX can measure many fluctuation/HBT signals as well as identify charged particles at low $p_{T}$ and neutral pions at high $p_{T}$. One of the biggest advantage that PHENIX has is a large acceptance for leptons and photons in the central arms that can scope rare leptonic and photonic observables such as dielectron continuum and hadronic and non-hadronic photons. Here we discuss the feasibility of measuring these observables at lower $\sqrt{s}$.

\subsection{Nuclear modification factors of high $p_{T}$ hadrons}

Nuclear modification factor $\left(R_{A A}\right)$ of high $p_{T}$ hadrons (mainly $\pi^{0}$ in our case) has been of great interest in all the collision systems since we discovered the suppression of the hadron yield in $\mathrm{Au}+\mathrm{Au}$ collisions at $\sqrt{s_{N N}}=130 \mathrm{GeV}$ for the first time [8], which was later confirmed to be a consequence of an energy loss of hard scattered partons by a control experiment in $\mathrm{d}+\mathrm{Au}$ collisions [9] and direct photon measurement in $\mathrm{Au}+\mathrm{Au}$ collisions [10]. The excitation function of $R_{A A}$ of high $p_{T}$ hadrons would be directly connected to the parton (gluons or quarks) density of the matter created. It is expected that in a low energy system, the partonic degree of freedom vanishes and thus the energy loss of partons disappears. At SPS energy $\left(\sqrt{s_{N N}}=17.3 \mathrm{GeV}\right)$, it was understood that the Cronin effect (initial multiple scattering of hard partons) dominates the nucleus-nucleus collisions [11]. However, by taking a ratio of $\mathrm{A}+\mathrm{A}$ to $\mathrm{p}+\mathrm{A}$, it was found that there is a suppression of hadron yield even at SPS [12]. The result from $\mathrm{p}+\mathrm{A}$ collisions was assumed to be a measure of the cold nuclear matter effect like Cronin, therefore the $\mathrm{A}+\mathrm{A} / \mathrm{p}+\mathrm{A}$ result indicated that the energy loss of hard partons had happened even at this low energy. At RHIC, PHENIX has measured $R_{A A}$ of $\pi^{0}$ 's as a function of collision energies in $\mathrm{Cu}+\mathrm{Cu}$ collisions as shown in Fig. 5 [13]. At $22.4 \mathrm{GeV}$,
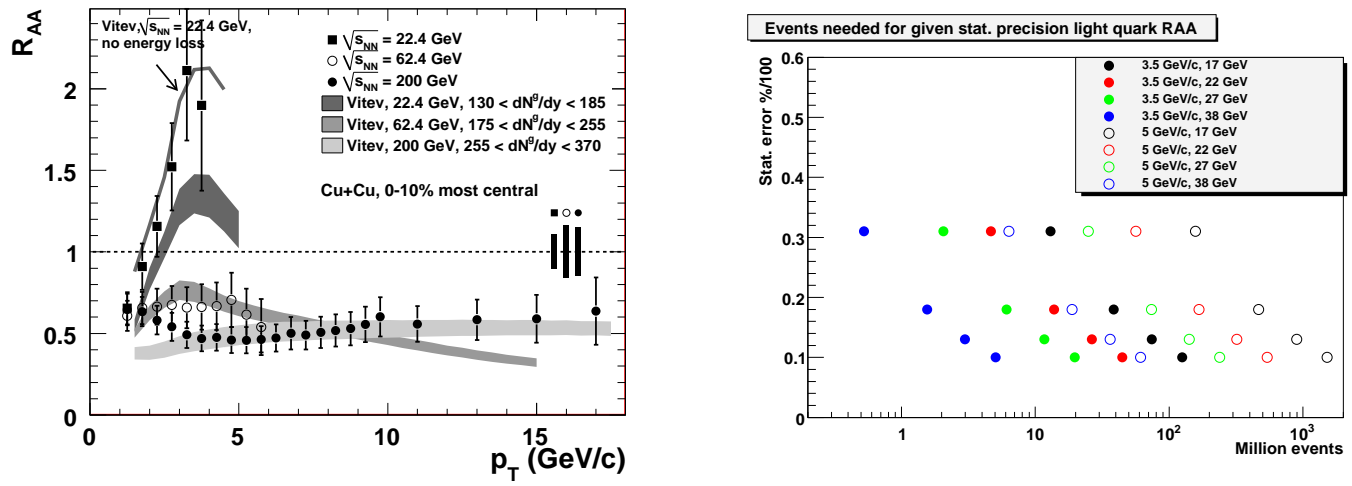

Figure 5: Left: $\pi^{0} R_{A A}$ in minimum bias $\mathrm{Cu}+\mathrm{Cu}$ collisions as a function of $\sqrt{s_{N N}}$, performed by the PHENIX experiment. Right: significance of signal as a function of number of events recorded, at several $\sqrt{s_{N N}}$ points.

the Cronin-like enhancement is seen, while at $62.4 \mathrm{GeV}$ and $200 \mathrm{GeV}$, suppression is dominant. For $22.4 \mathrm{GeV}$, unfortunately, $\mathrm{p}+\mathrm{p}$ reference data were not taken. Therefore, a complicated averaging of data taken by other experiments had to be carried out to obtain the reference. In order to fully accomplish $22.4 \mathrm{GeV}$ program, we need to measure hadron yields in $\mathrm{p}+\mathrm{p}$ collisions. It is one of the most urgent tasks in a low energy running. In order to quantify the nuclear effect at $22.4 \mathrm{GeV}$, we may want to take $\mathrm{d}+\mathrm{A}$ data as well. We estimated the number of events needed to measure 
$R_{A A}$ with sufficient significance for $\sqrt{s}=17-38 \mathrm{GeV}$, as shown in Fig. 5 . We show the machine time needed to accumulate the events, later in this paper.

\subsection{Non-hadronic photon measurement}

Electro magnetic probes such as photons or leptons have a big advantage over other observables in the sense that they are produced from all the collisional stages and don't interact with medium once produced. Therefore, by measuring the yields of the probes as a function of $p_{T}$ and/or mass, we can obtain quantities related to thermodynamical states of the collisional stages where photons or leptons are produced. The first significant result on non-hadronic photons (photons not from hadron decays) was obtained by the WA98 experiment [14]. The result was analyzed by theorists to obtain the temperature of the system produced. One of the many attempts is shown in Fig. 6 [15]. The issue in analyzing the data was that there was no measurement of hard direct
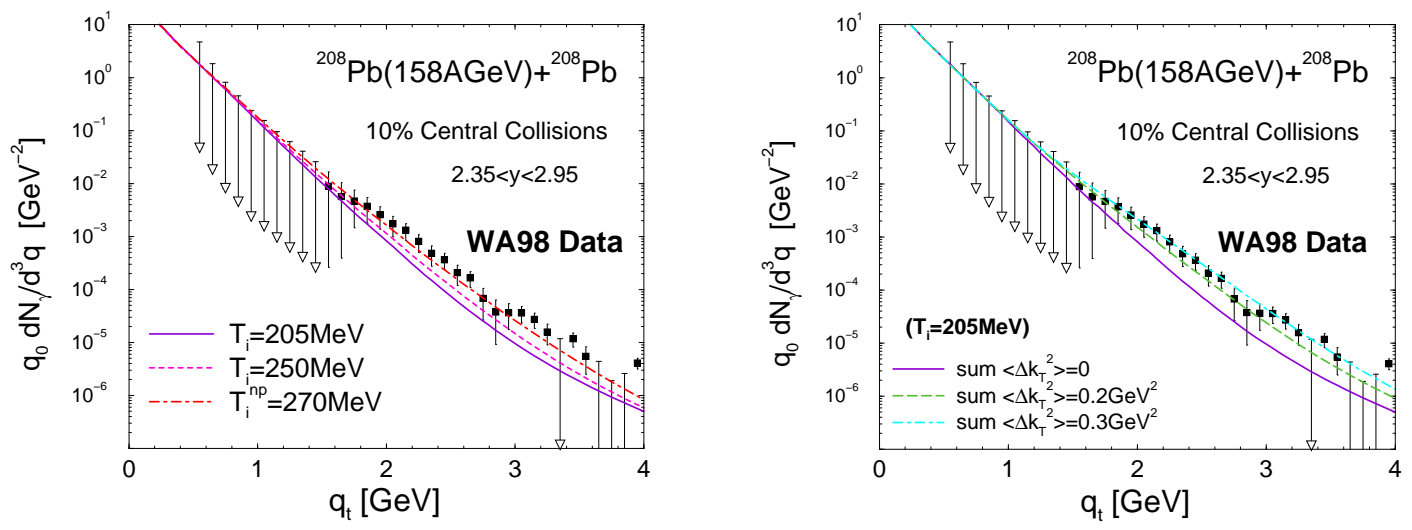

Figure 6: WA98 results and comparison with theoretical calculation with various initial temperatures $\left(T_{i}\right)$ and initial momentum broadenings $\left(<k_{T}>\right)$.

photons (produced in initial hard scattering). Therefore, the ratio of thermal photons and hard photons could not be determined, and the data allowed many degrees of freedom in the theory calculation. Had the $p_{T}$ range been extended to higher $p_{T}$ and confirmed the contribution of hard photons, the initial temperature would have been determined without large ambiguity. After years, WA98 has shown a preliminary result of direct photons in $\mathrm{p}+\mathrm{Pb}$ system [16]. The result does not have significant signal, and therefore would not be a measure of initial effect.

The PHENIX experiment has measured hard direct photons in 2002 for the first time in relativistic heavy ion collisions [10] as shown in Fig. 7. It proved that the initial hard scattering was not suppressed and the suppression of high $p_{T}$ hadrons was due to a final state effect. The measurement of high $p_{T}$ photons is essential to establish the baseline related to hard probes.

For the low $p_{T}$, the signal is obscured by the large background of $\pi^{0}$ and $\eta$ decays, whose yield is much higher than the expected signal of thermal photons. A technique for dielectron measurement is applied, assuming that the low mass high $p_{T}$ dielectrons are essentially the virtual photons that are produced (although with much smaller probability) in the same process of as that produce real photons. By selecting the mass region of $m_{\pi}<m_{e e}<<p_{T}$, one can eliminate a large background from $\pi^{0}$ Dalitz decays [17]. The dielectron yield is then converted to real photon yield using Kroll-Wada formula [18]. The data obtained with this method is shown in Fig. 7. The signal 

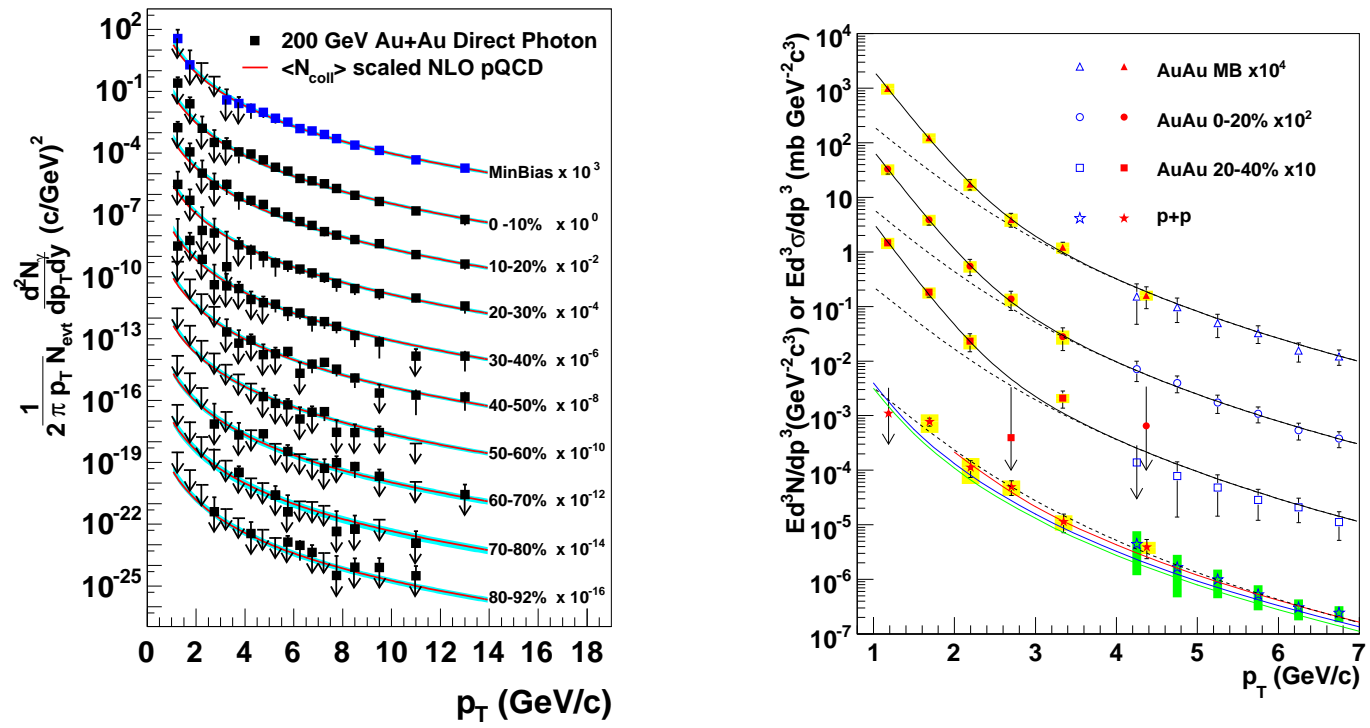

Figure 7: High $p_{T}$ hard photon (left) and low $p_{T}$ photon (right) measurement by PHENIX.

to background ratio will be much more improved by adding information from the hadron blind detector (HBD), which was installed in PHENIX last year (Fig. 8).

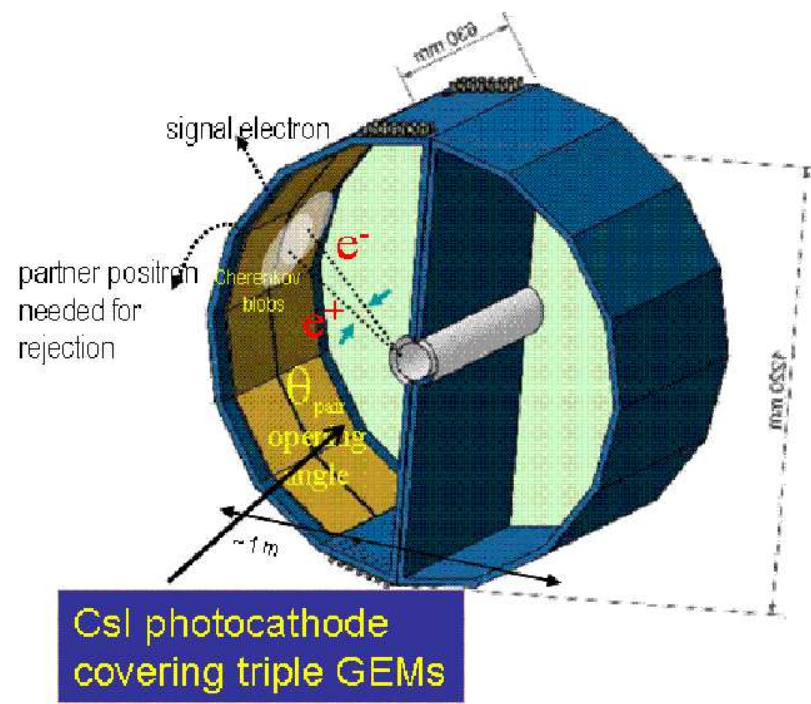

Figure 8: Hadron blind detector (HBD) to tag Dalitz decay electrons from $\pi^{0}$ and conversion electrons.

\subsection{Di-lepton measurement}

Di-electrons have more degree of freedom in terms of slicing collisional stages as well as efficiently eliminating backgrounds. The HBD detector in PHENIX can tag Dalitz decay electrons from $\pi^{0}$ as well as electron pairs produced via conversion of photons at beam pipe [7]. The HBD 
is a Cherenkov detector consisting of $\mathrm{CF}_{4}$ gas radiator, photo-cathodes and three stage gas electron multipliers (GEMs), and will be operated in a field free region. The electrons from $\pi^{0}$ Dalitz and photon conversions will have small opening angle which will form a single cluster with higher charge (corresponding to Cherenkov photo-electrons for two electrons), while single electron will form a cluster with lower charge (i.e., Cherenkov photo-electrons for one electron). The HBD detector will be kept installed till the end of 2010, when a new detector, VTX detector, is going to be installed to the same position to identify electrons from heavy flavor decays. Therefore, these two years will be a golden opportunity for PHENIX to measure dielectrons in heavy ion collisions. Fig. 9 shows the possibility of measuring dielectrons with $50 \mathrm{M}$ events at $\sqrt{s_{N N}}=17 \mathrm{GeV}$ with and without HBD. This shows the measurement in feasible as low as $17 \mathrm{GeV}$. The significance of the
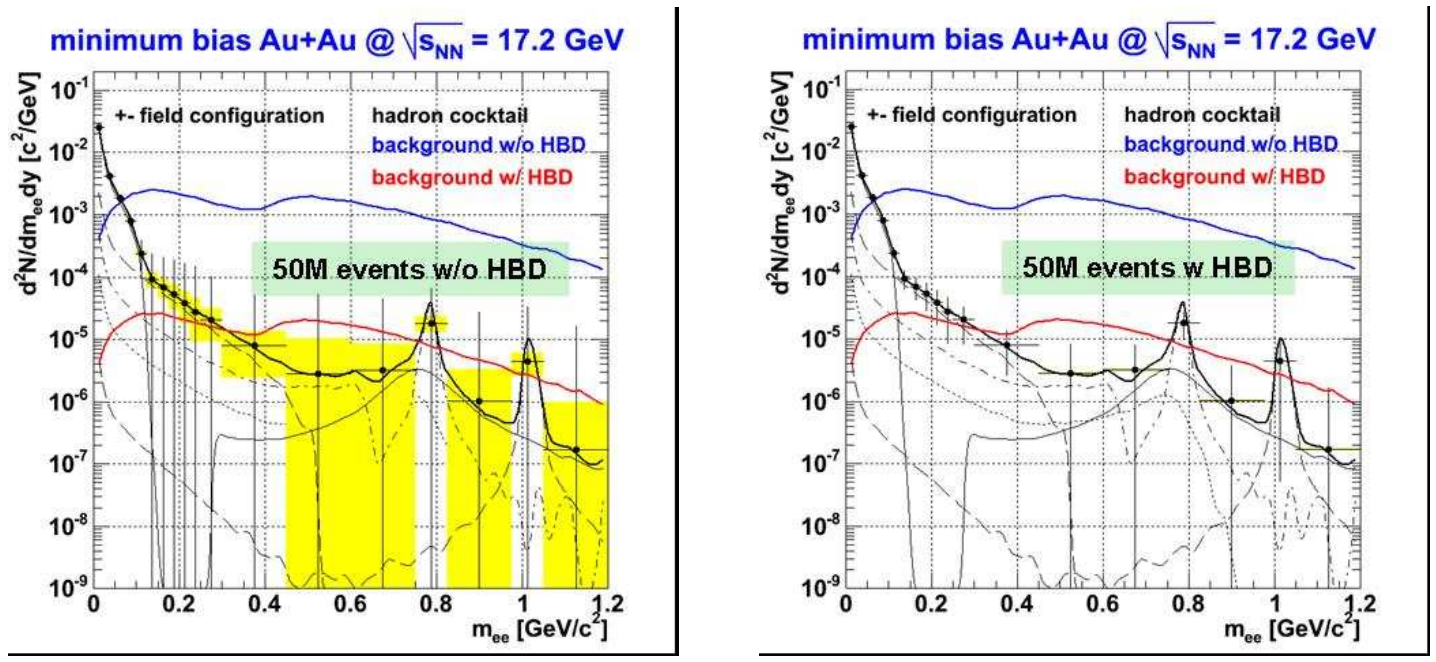

Figure 9: Di-electron measurement without (left) and with (right) $\mathrm{HBD}$ at $\sqrt{s_{N N}}=17 \mathrm{GeV}$. The statistical errors are significantly reduced because the background Dalitz or photon conversion electrons are suppressed by the HBD.

signal improves at higher energy. Before the trigger upgrades, the PHENIX detector is less efficient in triggering at sub-injection energies, but with the HBD, has a big advantage of measuring such rare probes as dielectrons. Therefore, PHENIX may want to have a beam at higher energies next year.

\section{Event rates}

The estimated number of minimum bias $\mathrm{Au}+\mathrm{Au}$ collisions to make a significant measurement with the PHENIX detector for each of the observables considered is summarized in Table 1.

A rate estimate was performed based upon the rates and efficiencies observed in the $9 \mathrm{GeV}$ $\mathrm{Au}+\mathrm{Au}$ test run and the corresponding $19.6 \mathrm{GeV}$ and $62.4 \mathrm{GeV}$ data. Peak luminosity projections for 2011 were assumed. Below injection energy an improved luminosity of a factor of 6.0 was assumed [19]. The main source of this improvement comes from filling all available bunches. In the Run-9 9.2 GeV Au+Au data analysis, a significant fraction of bad events was found, likely from beam-gas collisions and interactions with the beam pipe. Simply requiring a reconstructed BBC z-vertex was not sufficient to isolate good collisions. The additional requirement of a reconstructed 


\begin{tabular}{|c|c|c|c|c|c|c|c|c|c|c|c|c|c|c|c|c|c|}
\hline $\mid$ & 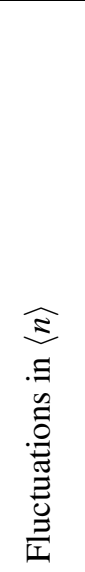 & 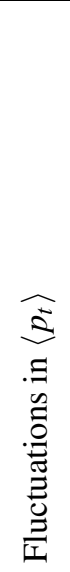 & 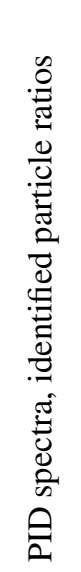 & 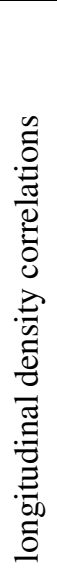 & 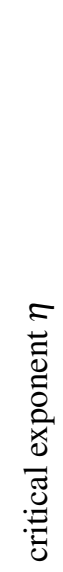 & 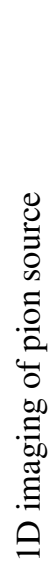 & 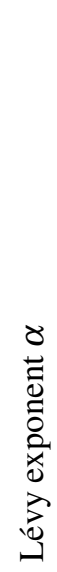 & 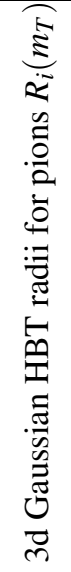 & 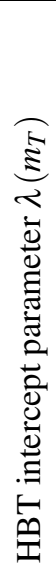 & 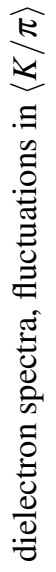 & 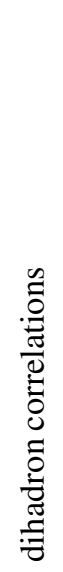 & 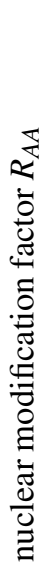 & 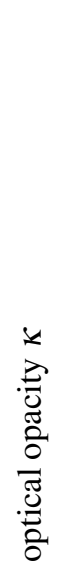 & 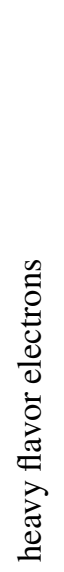 & 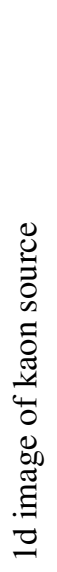 & 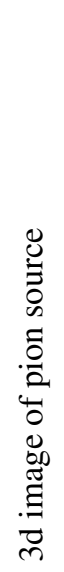 & 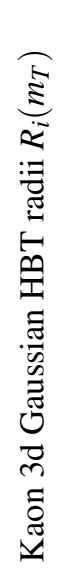 \\
\hline 5.5 & 0.01 & 0.03 & 0.03 & & 2 & \multicolumn{4}{|c|}{54} & 50 & 375 & \multicolumn{2}{|c|}{ NA } & $\overline{\mathrm{NA}}$ & \multicolumn{3}{|c|}{953} \\
\hline 7.7 & 0.01 & 0.03 & 0.02 & & 2 & \multicolumn{4}{|c|}{33} & 50 & 246 & \multicolumn{2}{|c|}{ NA } & $\overline{\mathrm{NA}}$ & \multicolumn{3}{|c|}{586} \\
\hline 11.5 & 0.01 & 0.03 & 0.02 & & 2 & \multicolumn{4}{|c|}{24} & 50 & 160 & \multicolumn{2}{|c|}{ NA } & $\overline{\mathrm{NA}}$ & \multicolumn{3}{|c|}{431} \\
\hline 17.3 & 0.01 & 0.03 & 0.01 & & 2 & \multicolumn{4}{|c|}{19} & 50 & 109 & \multicolumn{2}{|c|}{157} & $\overline{\mathrm{NA}}$ & \multicolumn{3}{|c|}{340} \\
\hline 27 & 0.01 & 0.03 & 0.01 & & 2 & \multicolumn{4}{|c|}{16} & 50 & 68 & \multicolumn{2}{|c|}{24} & NA & \multicolumn{3}{|c|}{276} \\
\hline 39 & 0.01 & 0.03 & 0.01 & & 2 & \multicolumn{4}{|c|}{14} & 50 & 48 & \multicolumn{2}{|c|}{6.3} & 700 & \multicolumn{3}{|c|}{239} \\
\hline
\end{tabular}

Table 1: Number of million Au+Au events needed to make a significant measurement with the PHENIX detector at various energies in the RHIC Low Energy Scan program.

\begin{tabular}{ccc}
$\sqrt{s_{N N}}$ & Minimum 10-hour-days & Maximum 10-hour-days \\
\hline 5.0 & 14.3 & 3714 \\
7.7 & 6.9 & 623.4 \\
11.5 & 3.5 & 119.0 \\
17.3 & 1.8 & 22.0 \\
27.0 & 0.4 & 2.5 \\
39.0 & 0.1 & 0.4
\end{tabular}

Table 2: Time estimates for accumulating $1 \mathrm{M}$ events based only upon the rates and efficiencies observed in the $9 \mathrm{GeV} \mathrm{Au+Au}$ test run with an improved luminosity of a factor of 6.0 (Maximum). Time estimates based upon the rates the assumption that the luminosity can be improved by a factor of 1.8 by 2012 and that the luminosity below injection energy improves by a factor of 6 along with the assumption that the recorded data purity is $100 \%$ (Minimum).

ZDC z-vertex is necessary to obtain a clean sample. However, at such low energies the fraction of the neutral particles that generates hits in the ZDC reduces drastically. Correspondingly, the required inclusion of the ZDC in the event cuts is found to be the primary reason that only 3\% of the BBCLL1 triggered events were available for analysis. Any improvement in the PHENIX triggering capabilities that does not require a coincidence with the ZDC would greatly improve the event purity at low energies and thus greatly decrease the amount of time necessary to achieve sufficient statistics for the observables of interest. This is demonstrated by Table 2 which show running time estimates for best and worst cases. In this estimate, we used HIJING as an event generator, which does not include Fermi motion effect. A preliminary study using UrQMD shows 
that the trigger efficiency at $\sqrt{s_{N N}}<10 \mathrm{GeV}$ is much higher than the one we found using HIJING. A further detailed study is ongoing.

\section{Summary}

PHENIX plans for low energy running are presented. Current detector setting makes it possible to measure dielectron spectra down to $\sqrt{s_{N N}}=39 \mathrm{GeV}$, and photon/high $p_{T}$ hadron spectra down to below sub-injection energy $\left(\sqrt{s_{N N}}=5-10 \mathrm{GeV}\right)$. A further study of trigger efficiency is ongoing using the UrQMD event generator. The upgrade of the trigger scheme after the installation of VTX detector will enable PHENIX to fully explore the sub-injection energy regime, starting 2011.

\section{References}

[1] K. Adcox et al. [PHENIX Collaboration], Nucl. Phys. A 757, 184 (2005).

[2] I. Arsene et al. [BRAHMS Collaboration], Nucl. Phys. A 757, 1 (2005).

[3] B. B. Back et al. [PHOBOS Collaboration], Nucl. Phys. A 757, 28 (2005).

[4] J. Adams et al. [STAR Collaboration], Nucl. Phys. A 757, 102 (2005).

[5] B. Alver et al. [PHOBOS Collaboration], Phys. Rev. Lett. 102, 142301 (2009).

[6] K. Adcox et al. [PHENIX Collaboration], Nucl. Instrum. Meth. A 499, 469 (2003).

[7] Z. Fraenkel et al., Nucl. Instrum. Meth. A 546, 466 (2005).

[8] K. Adcox et al. [PHENIX Collaboration], Phys. Rev. Lett. 88, 022301 (2002).

[9] S. S. Adler et al. [PHENIX Collaboration], Phys. Rev. Lett. 91, 072303 (2003).

[10] S. S. Adler et al. [PHENIX Collaboration], Phys. Rev. Lett. 94, 232301 (2005).

[11] E. Wang and X. N. Wang, Phys. Rev. C 64, 034901 (2001) and references therein.

[12] M. M. Aggarwal et al. [WA98 Collaboration], Phys. Rev. Lett. 100, 242301 (2008).

[13] A. Adare et al. [PHENIX Collaboration], Phys. Rev. Lett. 101, 162301 (2008).

[14] M. M. Aggarwal et al. [WA98 Collaboration], Phys. Rev. Lett. 85, 3595 (2000).

[15] S. Turbide, R. Rapp and C. Gale, Phys. Rev. C 69, 014903 (2004).

[16] C. Baumann [WA98 Collaboration], J. Phys. G 35, 104123 (2008).

[17] A. Adare et al. [PHENIX Collaboration], arXiv:0804.4168 [nucl-ex].

[18] N. M. Kroll and W. Wada, Phys. Rev. 98, 1355 (1955).

[19] private communication by Todd Satogata. 